Rabaska

Revue d'ethnologie de l'Amérique française

\title{
Centre de recherche sur l'histoire et le patrimoine de Charlevoix
}

\section{Serge Gauthier et Christian Harvey}

Volume 11, 2013

URI : https://id.erudit.org/iderudit/1018562ar

DOI : https://doi.org/10.7202/1018562ar

Aller au sommaire du numéro

Éditeur(s)

Société québécoise d'ethnologie

ISSN

1703-7433 (imprimé)

1916-7350 (numérique)

Découvrir la revue

Citer ce document

Gauthier, S. \& Harvey, C. (2013). Centre de recherche sur l'histoire et le patrimoine de Charlevoix. Rabaska, 11, 288-289.

https://doi.org/10.7202/1018562ar d'utilisation que vous pouvez consulter en ligne.

https://apropos.erudit.org/fr/usagers/politique-dutilisation/ 


\section{QUÉBEC}

\section{Centre de recherche sur l'histoire et le patrimoine de Charlevoix}

156, de l'Église

La Malbaie (Québec) G5A 1R4
Téléphone : (418) 665-8159

Courriel : shdc@sympatico.ca

Toile : www.shistoirecharlevoix.com

Le CRHPC vise à favoriser et à diffuser la recherche concernant l'histoire et le patrimoine de Charlevoix. Plusieurs chantiers ont été ouverts au cours de l'année.

\section{IDENTITÉ MÉTISSE}

Serge Gauthier, chercheur de notre Centre, a été retenu comme témoin expert dans le cadre de la Cause Corneau visant à établir la reconnaissance de l'identité métisse de la Communauté métisse du Domaine du Roi et de la Seigneurie de Mingan (CMDRSM). Il a ainsi rédigé une contre-expertise concernant des études déposées par le Procureur général du Québec comprenant près de 120 pages et qui devient une pièce liée à cette cause juridique. Christian Harvey, aussi de notre Centre, a préparé pour la même communauté métisse un document sur les critères du Rapport Powley en lien avec les recherches de l'historien Russel Bouchard et celles du chercheur Ser-Alexander Alemann qui sert aussi à la cause juridique. Serge Gauthier et Christian Harvey ont présenté deux conférences sur la question métisse dans Charlevoix (une en mai 2012 et une autre en mai 2013) devant l'assemblée générale du Clan Métis de Charlevoix. Un numéro de la Revue d'histoire de Charlevoix (numéro 71, juin 2012) a aussi été publié par notre équipe de recherche sur la présence autochtone dans Charlevoix.

\section{ART ET PEINTURE POPULAIRES}

En septembre 2011, à l'occasion d'une exposition de peintures consacrée aux peintres populaires de Charlevoix, un texte de Serge Gauthier intitulé « Blanche Bolduc et les peintres populaires de Charlevoix » a été publié par la Galerie d'art Walter Klinkhoff de Montréal. Ce texte est toujours accessible sur internet : www.Klinkhoff.com. Le même texte a été diffusé dans un numéro de la Revue d'histoire de Charlevoix (numéro 73, février 2013) consacré à la présence de peintres canadiens majeurs dans le village de Saint-Urbain.

Dès l'été 2013, Serge Gauthier va rédiger un texte sur le peintre Robert Cauchon originaire de La Malbaie en lien avec une exposition des œuvres de ce peintre charlevoisien qui se tiendra à l'hôtel de ville de Clermont où a aussi vécu cet artiste. Les recherches se poursuivent donc sur cette importante question de l'art et de la peinture populaire dans Charlevoix.

Histoire de la milice de CharlevoiX et numérisation des archives du RÉGiment deS Voltigeurs De QuéBeC

Les Éditions Charlevoix dirigée par notre Centre et la Société d'histoire de Charlevoix publieront à l'automne 2013 une imposante recherche menée par l'historien militaire Raymond Falardeau sur la milice dans Charlevoix. Cette parution va traiter 
d'un sujet inédit et s'annonce déjà comme un livre d'importance. Notre centre de recherche, grâce à l'expertise de Christian Harvey en ce domaine, s'occupe aussi de la numérisation de nombreuses pièces d'archives du Musée des Voltigeurs de Québec depuis le printemps 2013, en plus de voir aussi à numériser les photos anciennes de la collection de la Société d'histoire de Charlevoix.

\section{ENCYCLOPÉDIE DU PATRIMOINE CULTUREL DE L'AMÉRIQUe FRANÇAISE}

Les chercheurs de notre Centre ont eu une production impressionnante dans le cadre de l'Encyclopédie du patrimoine culturel de l'Amérique française (www.ameriquefrancaise.org). Serge Gauthier a ainsi publié douze textes sur ce site sur les sujets suivants : Alexis le Trotteur, Arthur Villeneuve, le Ô Canada, le Temps des sucres au Québec, la Chasse à l'orignal, les Ramancheurs au Québec, Paul Provencher, Menaud maître-draveur, le Domaine Forget, la Gourgane, le peintre Claude le Sauteur et le Cirque du Soleil. Les quatre derniers textes furent rédigés en collaboration avec Christian Harvey. Celui-ci a aussi publié sur les thèmes suivants : le Barrage DanielJohnson, Jack Kérouac, le Séminaire de Québec, la Vieille prison de Trois-Rivières.

\section{EXPOSITION PERMANENTE}

La Société d'histoire de Charlevoix a conduit la recherche historique pour le site internet de la nouvelle exposition permanente du Musée de Charlevoix (Section histoire régionale) inaugurée en janvier 2012. Christian Harvey fut le chargé de projet de cette belle réalisation.

\section{ARCHIVES DU CRHPC}

La Société d'histoire de Charlevoix a remis la propriété de ses archives au Centre de recherche sur l'histoire et le patrimoine de Charlevoix. L'organisme compte 48 fonds, 26 collections, et divers dossiers correspondant à 50,75 mètres linéaires d'archives. Plus de 10000 photos historiques et près de 1000 livres complètent notre documentation sur l'histoire régionale de Charlevoix. Ce service d'archives est accessible sur rendez-vous seulement.

Trois nouveaux instruments de recherche ont été produits récemment grâce à la participation de l'étudiant en histoire Jean-Benoît Guérin-Dubé, soit : le Catalogue de dépôt du centre d'archives de la Société d'histoire de Charlevoix (2011) ; la Bibliographie des titres parus sur la région de Charlevoix entre 1996 et 2012 ; l'instrument de recherche du fonds Jacques Barnard (abbé). 